Original research article

\title{
Ovulation inhibition with four variations of a four-phasic estradiol valerate/dienogest combined oral contraceptive: results of two prospective, randomized, open-label studies 2 , 弥访
}

\author{
Jan Endrikat ${ }^{\mathrm{a}, \mathrm{b}, *}$, Susanne Parke ${ }^{\mathrm{c}}$, Dietmar Trummer ${ }^{\mathrm{c}}$, Werner Schmidt ${ }^{\mathrm{b}}$, \\ Ingrid Duijkers ${ }^{\mathrm{d}}$, Christine Klipping ${ }^{\mathrm{d}}$ \\ ${ }^{a}$ Bayer, Inc., Toronto, Ontario M9W 1G6, Canada \\ ${ }^{\mathrm{b}}$ Universitätskliniken des Saarlandes, Frauenklinik, 66421 Homburg/Saar, Germany \\ ${ }^{\mathrm{c}}$ Bayer Schering Pharma AG, D-13342 Berlin, Germany \\ ${ }^{\mathrm{d}}$ Dinox BV, NL-9713 GZ Groningen, The Netherlands \\ Received 31 July 2007; revised 19 April 2008; accepted 6 May 2008
}

\begin{abstract}
Background: Attempts to improve the tolerability of combined oral contraceptives (COCs) have included the substitution of ethinylestradiol (EE) with 17ß-estradiol (E2). However, this has proved unsatisfactory, specifically in terms of cycle control. To improve upon the poor cycle control seen previously, E2 [in the form of estradiol valerate (E2V); $1 \mathrm{mg}$ of E2V contains $0.76 \mathrm{mg}$ of E2] was combined with dienogest (DNG) in a novel four-phasic regimen. In the current studies, the ovulation-inhibition potency of four variations of this regimen was assessed. Study Design: Two randomized, open-label, Phase II studies were performed. The first study compared two regimens (Regimens 1A and 2A) with similar dosages of DNG but different lengths of application. Having established in Study 1 that the length of application of Regimen 2A was most suitable, but that the dosages of DNG were too low for effective ovulation inhibition, a second study, which compared two regimens (Regimens $2 \mathrm{~B}$ and $2 \mathrm{C}$ ) with similar lengths of application but with increased dosages of DNG, was undertaken. The primary efficacy variable in both studies was the proportion of women with a Hoogland score of 5 or 6 during Cycle 2.

Results: The full analysis set comprised 192 and 203 women in Studies 1 and 2, respectively. In Study 1, 10 women (10.9\%) in Regimen 1A and 6 women (6.4\%) in Regimen 2A had a Hoogland score of 5 or 6. In Study 2, three women (3.1\%) in Regimen 2B and one woman (1.0\%) in Regimen $2 \mathrm{C}$ had a Hoogland score of 5 or 6 . There were no safety concerns with any of the regimens.

Conclusion: The results of these studies identified a four-phasic COC preparation comprising E2V/DNG that provides efficient ovulation inhibition. It is expected that this regimen will lead to an innovative COC containing E2 instead of EE.
\end{abstract}

(C) 2008 Elsevier Inc. All rights reserved.

Keywords: Ovulation inhibition; Four-phasic regimen; Estradiol valerate; Dienogest

is This study was supported by an unrestricted grant from Bayer Schering Pharma AG (Berlin, Germany).

论 Jan Endrikat, Susanne Parke, and Dietmar Trummer are employees of Bayer Schering Pharma AG. Ingrid Duijkers and Christine Klipping are employees of the Contract Research Organisation Dinox BV who were contracted to perform the studies.

* Corresponding author. Bayer, Inc., Toronto, Ontario, Ontario M9W 1G6, Canada. Tel.: +1 416246 5033; fax: +1 4162489526 .

E-mail address: jan.endrikat.b@bayer.com (J. Endrikat).

\section{Introduction}

There have been several attempts in the past to increase the tolerability of combined oral contraceptives (COCs). Such initiatives have included introducing new progestins [1-3], reducing the dose of ethinylestradiol (EE) [4-7] and replacing EE with 17ß-estradiol (E2) [8-19]. However, previous attempts to replace EE with E2 proved somewhat unsatisfactory with respect to cycle control $[8,13,15-17]$, particularly when E2 was administered as part of a monophasic or a biphasic regimen. 
In order to improve upon the poor cycle control observed in previous studies of E2-containing COCs, a novel preparation was developed in which E2 [in the form of estradiol valerate (E2V); $1 \mathrm{mg}$ of E2V contains $0.76 \mathrm{mg}$ of E2] was combined with dienogest (DNG) in a four-phasic regimen [14,19]. DNG was included as the progestin component, as it is known to have a particularly strong endometrial effect [20]. A unique four-phasic regimen, which incorporated an estrogen step-down and a progestin step-up, was used to solve the problem of poor cycle control observed with previous E2-containing COCs. Early estrogenic dominance with the regimen ensured initial endometrial proliferation and sensitivity to midcyclic progestin action, while the use of DNG, with its specific endometrial affinity and dominance during the middle part to the late part of the cycle, ensured endometrial stroma stability, particularly towards the end of the cycle.

In the current studies, the ovulation-inhibition potency of four variations of the four-phasic preparation of E2V and DNG described above was assessed. The purpose of these studies, which were performed in an iterative stepwise manner, was to determine the optimal length of application (Study 1) and the required dose of DNG (Study 2) for effective inhibition of ovulation.

\section{Materials and methods}

\subsection{Study design}

Two prospective, randomized, open-label studies were performed at two centers: one in Germany (Clinical Research, Dinox GmbH, Berlin, Germany) and another in The Netherlands (Dinox Medical Investigations, Nijmegen, The Netherlands). The two studies were performed in sequence, with each study investigating two regimens. In the first study, Regimens $1 \mathrm{~A}$ and $2 \mathrm{~A}$, which comprised the same dosages of DNG but different lengths of application, were investigated. In the second study, having established that the length of application of Regimen 2A was suitable but that the dosages of DNG were too low for effective ovulation inhibition, Regimens $2 \mathrm{~B}$ and $2 \mathrm{C}$ were investigated. Regimens $2 \mathrm{~B}$ and $2 \mathrm{C}$ comprised the same length of application as Regimen 2A, but included distinctly increased dosages of DNG. Fig. 1 shows each of the four regimens investigated across the two studies. All regimens applied E2V in doses decreasing from $3 \mathrm{mg}$ to $1 \mathrm{mg}$. Since the valerate group of E2V is split off during resorption and first-pass metabolism, this results in a total E2 dosage of $0.764 \mathrm{mg}$ per $1 \mathrm{mg}$ of E2V applied [21]. Previous research has shown that this is the lowest effective estrogen dosage, and that further lowering of the E2V dose results in reduced contraceptive efficacy (data on file; Bayer Schering Pharma AG, Berlin, Germany).

Both studies were conducted in accordance with the ethical principles of the Declaration of Helsinki and the International Conference on Harmonization-Good Clinical Practice Guidelines (January 17, 1997). The Ethics Commit- tees reviewed and gave their consent to the protocols before the studies started. All women gave their informed consent before participating in the study.

\subsection{Subjects}

Healthy women aged 18-35 years (smokers not older than 30 years) were eligible for inclusion in the studies. The exclusion criteria were established based on contraindications for COC use. Further exclusion criteria included specified coexisting diseases, including metabolic and endocrine diseases, diagnostically unclassified genital bleeding and a history of liver or vascular diseases. In addition, women using medications that could influence the study objectives, such as sex steroids and drugs known to induce or inhibit liver enzymes, were also excluded. Women had to be willing to use nonhormonal contraceptive methods to protect themselves from getting pregnant throughout the study. On screening, all women underwent thorough medical and gynecological examinations, including a cervical cytology examination using the Papanicolaou method and a pregnancy test. Eligible women underwent a pretreatment observation cycle (which started on the first day of menstruation following screening), and those who ovulated or had a follicular diameter of $\geq 15 \mathrm{~mm}$ on or before Day 23 of the observation cycle, as assessed by transvaginal ultrasound (TVU) examination (Siemens Sonoline Siena GM 6600A2E00, transvaginal probe $6.5 \mathrm{EV}^{+}{ }^{+}$ Convex Array Endo-V Probe or a Toshiba EccOcee model SSA-340A), were randomized to treatment.

\subsection{Study preparations}

The study medications (produced by Bayer Schering Pharma AG) were supplied in calendar packs. Treatment was received in consecutive cycles of 28 days with no pill-free interval. The treatment period was up to three cycles in Study 1 and also in Study 2. The first tablet was taken on the first day of menstrual bleeding. The tablets were to be taken at approximately the same time each day, with the interval between two tablets as close as possible to $24 \mathrm{~h}$. When the scheduled tablet intake time was missed, the women had to take the tablet as soon as they remembered, at the latest with the next tablet. Tablet intake was recorded in a diary on a daily basis.

Subjects were randomized to treatment in order of arrival at the treatment center. Randomization was performed at Bayer Schering Pharma AG using randomization blocks. Randomization numbers were equally distributed between the two treatment groups in each study.

\subsection{Study assessments}

\subsubsection{Efficacy}

Both studies were carried out to evaluate ovulation inhibition, which was assessed by TVU monitoring of follicle size and analysis of serum E2 and progesterone levels, and classified according to the 6-point scoring system of Hoogland and Skouby [22]. The primary efficacy 

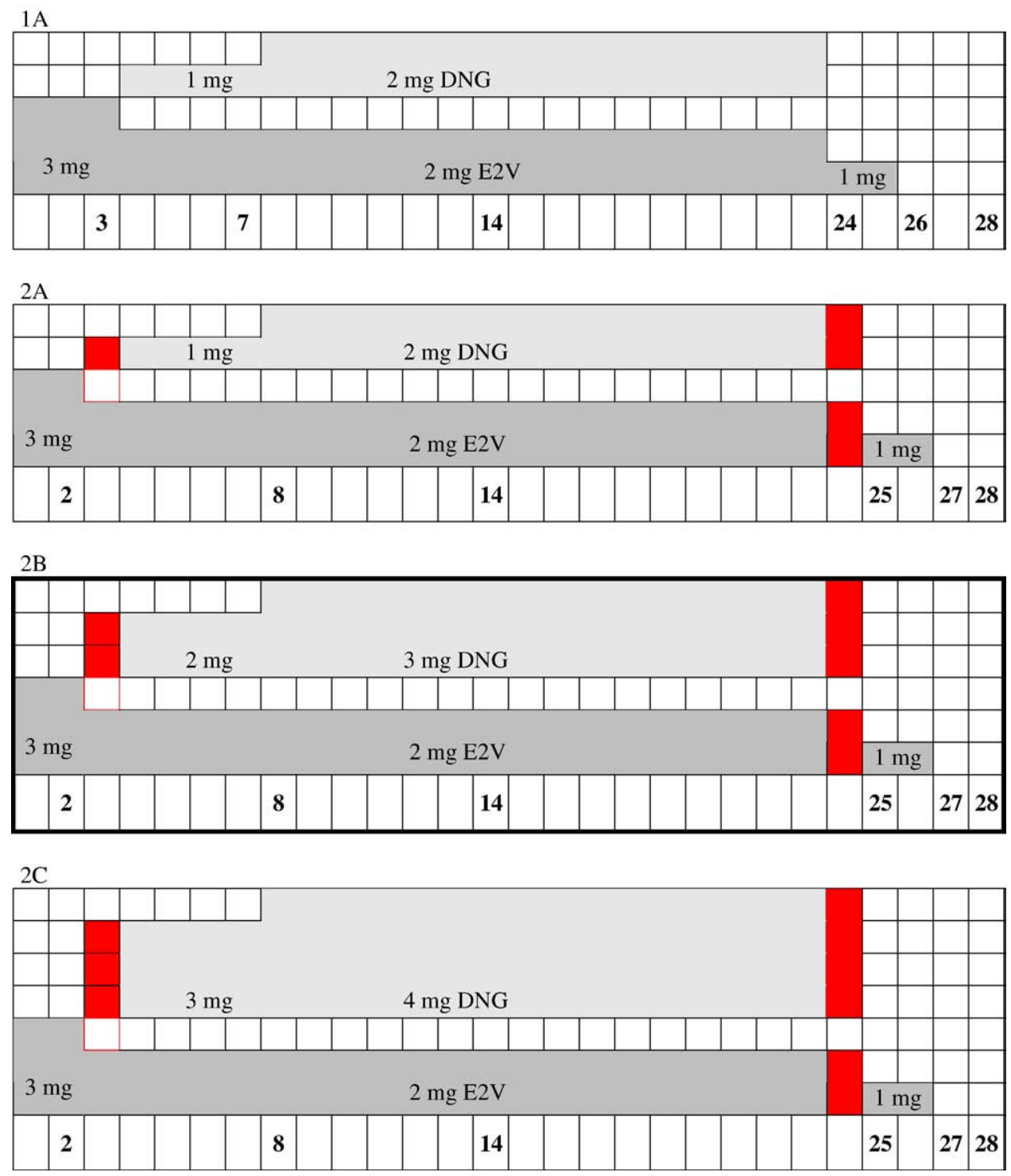

Fig. 1. Overview of four variations of a novel four-phasic COC containing E2V and DNG. Regimens $1 \mathrm{~A}$ and $2 \mathrm{~A}$ were initially investigated in Study 1 , while Regimens $2 \mathrm{~B}$ and 2C were subsequently investigated in Study 2. The modifications from Regimen $1 \mathrm{~A}$ to Regimens $2 \mathrm{~A}, 2 \mathrm{~B}$ and to $2 \mathrm{C}$ are highlighted.

end point in both studies was the proportion of women with a Hoogland score of 5 [luteinized unruptured follicle (LUF)] or a Hoogland score of 6 (ovulation) at Cycle 2. A Hoogland score of 6 is defined as a ruptured follicle-like structure (FLS) of $>13 \mathrm{~mm}$ with serum progesterone of $>5$ $\mathrm{nmol} / \mathrm{L}$ and $\mathrm{E} 2$ of $>0.1 \mathrm{nmol} / \mathrm{L}$. A Hoogland score of 5 was considered as critical as a Hoogland score of 6 , since a persisting FLS may still develop into a ruptured FLS in subsequent cycles. Other efficacy end points assessed in both studies included the proportion of women with a Hoogland score of 5 or 6 at Cycle 3 and the proportion of women with resumption of ovulation during the posttreatment cycle.

TVU examinations and blood sampling for E2 and progesterone were performed every third day ( \pm 1 day) in both studies. In each cycle, the highest levels of hormones and the greatest diameter of the follicle were used to calculate the Hoogland score. The hormone assessments were performed at a central laboratory in Germany (Laboratory for Clinical Research, LKF GmbH, Raisdorf, Germany) using assays based on the ElectroChemiLuminescense ImmunoAssay technology on the analyzer Elecsys (Roche Diagnostics GmbH, Mannheim, Germany).

In Study 1, TVU and hormone assessments were performed during an untreated precycle and during two treatment cycles. The precycle served to prove ovulation in all subjects, while Cycles 1 and 2 comprised the regular treatment cycles. Only those women with a nonpersisting FLS of $>13 \mathrm{~mm}$ (Hoogland score of 4 ) at the end of Cycle 2 were admitted to a third cycle of treatment in order to monitor 'stepwise' follicular growth. At one study center, all women not having a third cycle of treatment were monitored 
for one posttreatment cycle in order to assess the return of ovulation following treatment cessation.

Although the study procedures were identical in both studies, the focus of Study 2 was modified according to what was learned in Study 1. In Study 1, only Cycles 1 and 2 were intensively monitored, with Cycle 3 being assessed only in those women who showed a nonpersisting FLS of $>13 \mathrm{~mm}$ at the end of Cycle 2. This resulted in a high percentage of ovulations in Cycle 3. In order to clarify this phenomenon, the focus of Study 2 was modified so as to take data from Cycle 3 rather than from Cycle 1. As such, all subjects received treatment for three cycles, and study assessments were performed in the precycle and in Cycles 2 and 3, and not in Cycle 1. Similar to Study 1, one posttreatment cycle was monitored at one study center in Study 2 in order to assess the return of ovulation following treatment cessation.

\subsubsection{Safety}

Safety was monitored by recording adverse events, body weight, use of concomitant medications, treatment compliance, and routine safety and laboratory parameters.

\subsection{Statistical methods}

It was planned to recruit 200 healthy women in each study (100 women per treatment regimen). The sample size was not based on any power calculation. However, an a posteriori target was to reach an ovulation rate of $<5 \%$, with an upper $90 \%$ confidence limit of $10 \%$. After observing Hoogland scores of 5 or 6 in six women treated with Regimen $2 \mathrm{~A}$ in Study 1, it was expected that there would be up to four women with Hoogland scores of 5 or 6 with Regimens 2B and $2 \mathrm{C}$ in Study 2. Assuming a dropout rate of approximately $25 \%$, it was necessary to include 100 subjects per regimen into the study. All women who entered the studies and who took at least one tablet of study medication and for whom at least one observation after dosing was available were included in the full analysis set (FAS).

All efficacy variables and demographic and baseline variables were described according to their type, using descriptive statistics.

The probability of a Hoogland score of 5 or 6 in Cycle 2 was estimated by the relative frequency of women with a
Hoogland score of 5 or 6 . Two-sided $90 \%$ confidence intervals (CIs) were calculated for this probability for each treatment and each cycle using normal approximations:

$\hat{p}_{\mathrm{T}} \pm u_{0.95} \sqrt{\frac{\hat{p}_{\mathrm{T}}\left(1-\hat{p}_{\mathrm{T}}\right)}{n}}$

where subscript $\mathrm{T}$ denotes treatment, $\hat{p}_{\mathrm{T}}$ denotes the estimated proportion and $u_{0.95}$ denotes the $95 \%$ quantile of the normal distribution. In addition, all Hoogland scores were reported using descriptive statistics. All analyses were conducted using SAS software (version 8).

\section{Results}

\subsection{Study population}

In Study 1, a total of 236 women were screened, and 196 were randomized to treatment. Of these, 99 were assigned to Regimen 1A, and 97 were assigned to Regimen 2A. The FAS comprised 192 women (96 subjects in each of Regimens 1A and 2A). For the primary efficacy variable, data for Cycle 2 were evaluated in 92 and 94 women treated with Regimens $1 \mathrm{~A}$ and 2A, respectively. Thirty-one women with a nonpersisting FLS of $>13 \mathrm{~mm}$ at the end of Cycle 2 were admitted to a third treatment cycle.

In Study 2, a total of 268 women were screened, and 210 were randomized to treatment. Of these, 104 were assigned to Regimen 2B, and 106 were assigned to Regimen $2 \mathrm{C}$. The FAS comprised 203 women (100 subjects in Regimen 2B, and 103 subjects in Regimen 2C). For the primary efficacy variable, data for Cycle 2 were evaluated in 96 and 97 women treated with Regimens $2 \mathrm{~B}$ and $2 \mathrm{C}$, respectively.

The demographic and baseline characteristics were well matched in both studies and in all four treatment groups alike (Table 1).

\subsection{Primary efficacy variable}

In Study 1, 10 women (10.87\%; CI=5.53 16.21\%) treated with Regimen $1 \mathrm{~A}$ and six women $(6.38 \%$; $\mathrm{CI}=2.24-10.53 \%)$ treated with Regimen 2A had a Hoogland score of 5 or 6 in Cycle 2. In Study 2, three women (3.13\%; CI $=0.20-6.05 \%)$ treated with Regimen $2 \mathrm{~B}$ and one woman $(1.03 \%$; $\mathrm{CI}=0.00-$

Table 1

Demographic and baseline characteristics (FAS)

\begin{tabular}{|c|c|c|c|c|}
\hline & \multicolumn{2}{|l|}{ Study 1} & \multicolumn{2}{|l|}{ Study 2} \\
\hline & Regimen 1A $(n=96)$ & Regimen 2A $(n=96)$ & Regimen $2 \mathrm{~B}(n=100)$ & Regimen $2 \mathrm{C}(n=103)$ \\
\hline Age (years) $[$ mean \pm SD] & $25.4 \pm 4.1$ & $26.0 \pm 4.4$ & $25.6 \pm 3.7$ & $26.0 \pm 4.2$ \\
\hline Height $(\mathrm{cm})[$ mean $\pm \mathrm{SD}]$ & $167.8 \pm 6.2$ & $168.4 \pm 6.4$ & $169.3 \pm 6.5$ & $167.7 \pm 6.9$ \\
\hline Body weight $(\mathrm{kg})[$ mean $\pm \mathrm{SD}]$ & $63.6 \pm 9.9$ & $64.5 \pm 7.8$ & $63.9 \pm 7.4$ & $62.5 \pm 9.8$ \\
\hline Body mass index $\left(\mathrm{kg} / \mathrm{m}^{2}\right)[$ mean $\pm \mathrm{SD}]$ & $22.6 \pm 3.1$ & $22.8 \pm 2.7$ & $22.3 \pm 2.9$ & $22.2 \pm 2.8$ \\
\hline Nullipara $[n(\%)]$ & $77(80.2)$ & $72(75.0)$ & $89(89.0)$ & $82(79.6)$ \\
\hline Prevalence of current smoking $[n(\%)]$ & $34(35.4)$ & $35(36.5)$ & $33(33.0)$ & $35(34.0)$ \\
\hline $\begin{array}{l}\text { Oral contraceptive use before the start } \\
\text { of the study }[n(\%)]\end{array}$ & $43(44.8)$ & 39 (40.6) & $44(44.0)$ & $50(48.5)$ \\
\hline
\end{tabular}


$2.72 \%$ ) treated with Regimen $2 \mathrm{C}$ had a Hoogland score of 5 or 6 in Cycle 2 (Fig. 2). One of the six women in Group 2A with a Hoogland score of 5 or 6 had a LUF, while all others showed ovulations (Table 2).

\subsection{Other efficacy variables}

Of the 31 women in Study 1 who were admitted to a third treatment cycle, 8 of 15 women $(53.3 \%)$ treated with Regimen $1 \mathrm{~A}$ and 5 of 16 women (31.3\%) treated with Regimen 2A had ovulations in Cycle 3. In Study 2, two women $(2.2 \%)$ treated with Regimen $2 \mathrm{~B}$ and one subject $(1.05 \%)$ treated with Regimen $2 \mathrm{C}$ ovulated in Cycle 3.

Of the women assessed during a posttreatment cycle, more than $80 \%$ resumed ovulation (Table 2).

\subsection{Tolerability}

No safety concerns were noted with any of the regimens, and the majority of treatment-related adverse events were typical of those occurring during COC use (Table 3).

One woman in Study 1 (Regimen 1A) had a serious adverse event that was considered possibly treatmentrelated (ovarian cyst). No deaths occurred during the studies. Four women in Study 1 had adverse events considered possibly related to treatment that resulted in study discontinuation [Regimen 1A, $n=2$ (ovarian cyst, breast pain); Regimen 2A, $n=2$ (edema, diarrhea)]. In Study 2, eight women had treatment-related adverse events that resulted in discontinuation of treatment [Regimen 2B, $n=5$ (depression, headache, worsening acne, eye irritation, furunculosis); Regimen 2C, $n=3$ (emotional lability $(n=2)$, headache)].

\section{Discussion}

The two prospective, randomized, Phase II studies described here were performed to obtain information on the ovulation-inhibition potency of four variations of a novel four-phasic COC comprising E2V and DNG. The studies were carried out in a stepwise manner in order to determine, firstly, a suitable length of application (Study 1) and, secondly, a suitable dose of DNG (Study 2) for effective ovulation inhibition (Fig. 1). Each step increased the ovulation-inhibition potency remarkably, with the result that Regimen 2B was identified as the regimen with the lowest effective dose of DNG for efficient ovulation inhibition. This regimen - comprising $3 \mathrm{mg}$ of $\mathrm{E} 2 \mathrm{~V}$ for 2 days; $2 \mathrm{mg}$ of E2V/2 $\mathrm{mg}$ of DNG for 5 days; $2 \mathrm{mg}$ of E2V/3 $\mathrm{mg}$ of DNG for 17 days; $1 \mathrm{mg}$ of E2V for 2 days; and placebo for 2 days - contained the lowest dose of DNG that inhibited more than $95 \%$ of ovulations in Cycle 2.

COCs typically exhibit Pearl Indices ranging between 0 and 1 (pregnancies per 100 women-years) in clinical studies. Discrepancies between contraceptive efficacy in real-life conditions and the results of classical Pearl Index studies can, in part, be explained by the additional contraceptive effects provided by hormonal contraceptives through alterations in the cervical mucus and the endometrium [23]. Based on the pharmacodynamic properties of DNG [20], such extraovarian contraceptive effects are also expected with the combination of E2V and DNG.

As mentioned previously, because monitoring of Cycle 3 was only performed in those women who showed a nonpersisting FLS of $>13 \mathrm{~mm}$ at the end of Cycle 2 in

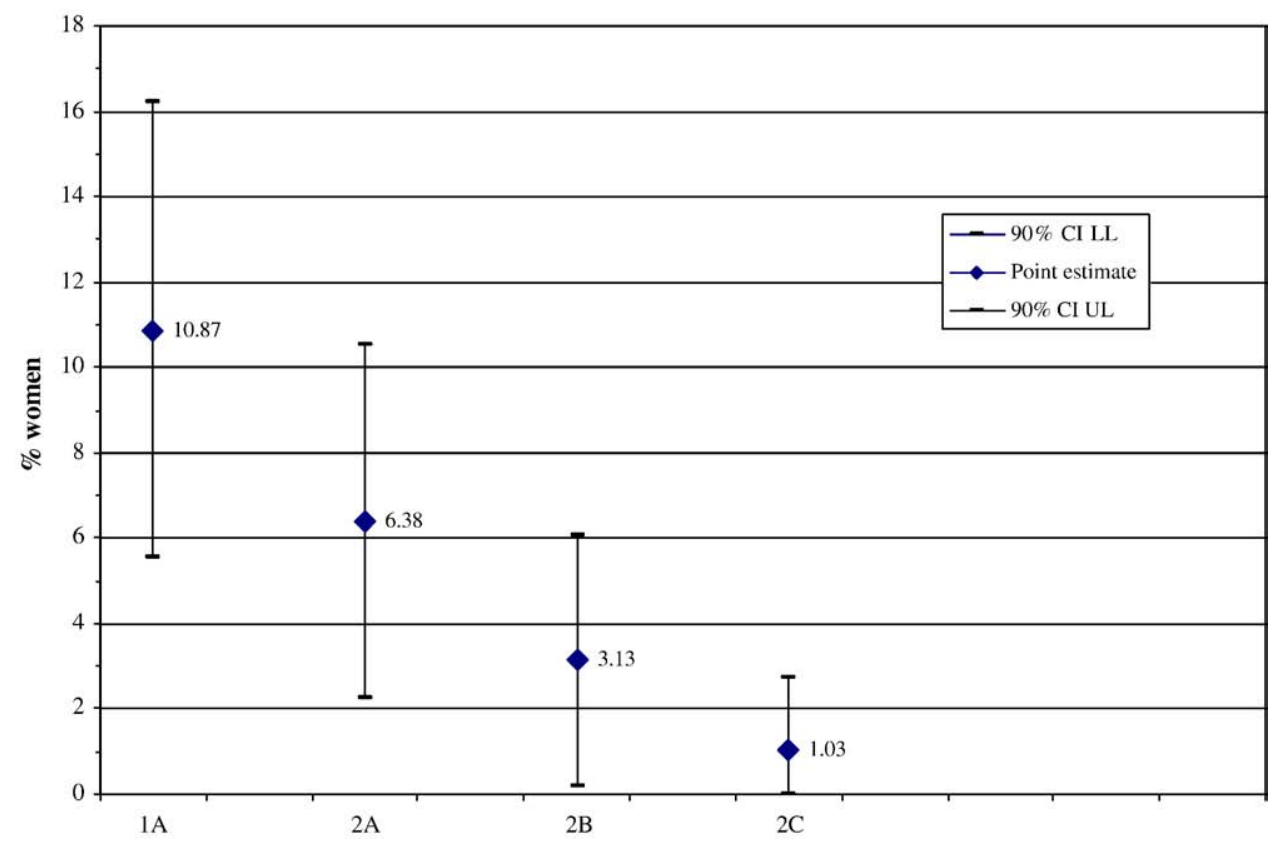

Fig. 2. Ovulation inhibition in Cycle 2: the percentage of women with a Hoogland score of 5 or 6 and $90 \%$ CIs (FAS). Regimens 1 A and 2A were initially investigated in Study 1, while Regimens 2B and 2C were subsequently investigated in Study 2. LL, lower limit; UL, upper limit. 
Table 2

Ovulation inhibition in Cycles 1-3 (FAS)

\begin{tabular}{|c|c|c|c|c|}
\hline \multirow[t]{2}{*}{ Hoogland score of 5 or $6[n(\%)]$} & \multicolumn{2}{|l|}{ Study 1} & \multicolumn{2}{|l|}{ Study 2} \\
\hline & Regimen 1A $(n=96)$ & Regimen 2A $(n=96)$ & Regimen 2B & Regimen $2 \mathrm{C}$ \\
\hline \multicolumn{5}{|l|}{ Cycle 1} \\
\hline LUF & $0(0.0)$ & $0(0.0)$ & ND & ND \\
\hline Ovulation & $0(0.0)$ & $0(0.0)$ & ND & ND \\
\hline \multirow[t]{2}{*}{ Hoogland score of 5 or $6[n(\%)]$} & \multicolumn{2}{|l|}{ Study 1} & \multicolumn{2}{|l|}{ Study 2} \\
\hline & Regimen $1 \mathrm{~A}(n=92)$ & Regimen 2A $(n=94)$ & Regimen 2B $(n=96)$ & Regimen $2 \mathrm{C}(n=97)$ \\
\hline \multicolumn{5}{|l|}{ Cycle 2 (see Fig. 2) } \\
\hline LUF & $0(0.0)$ & $1(1.1)$ & $0(0.0)$ & $0(0.0)$ \\
\hline Ovulation & $10(10.9)$ & $5(5.3)$ & $3(3.1)$ & $1(1.0)$ \\
\hline \multirow[t]{2}{*}{ Hoogland score of 5 or $6[n(\%)]$} & \multicolumn{2}{|l|}{ Study 1} & \multicolumn{2}{|l|}{ Study 2} \\
\hline & Regimen 1A $(n=15)$ & Regimen $2 \mathrm{~A}(n=16)$ & Regimen 2B $(n=91)$ & Regimen $2 \mathrm{C}(n=95)$ \\
\hline \multicolumn{5}{|l|}{ Cycle 3} \\
\hline LUF & $0(0.0)$ & $0(0.0)$ & $0(0.0)$ & $0(0.0)$ \\
\hline Ovulation & $8(53.3)$ & $5(31.3)$ & $2(2.2)$ & $1(1.1)$ \\
\hline \multirow[t]{2}{*}{ Hoogland score of 5 or $6[n(\%)]$} & \multicolumn{2}{|l|}{ Study 1} & \multicolumn{2}{|l|}{ Study 2} \\
\hline & Regimen 1A $(n=35)$ & Regimen 2A $(n=40)$ & Regimen 2B $(n=39)$ & Regimen $2 \mathrm{C}(n=44)$ \\
\hline \multicolumn{5}{|l|}{ Posttreatment cycle } \\
\hline LUF & $0(0.0)$ & $0(0.0)$ & $0(0.0)$ & $0(0.0)$ \\
\hline Ovulation & $30(85.7)$ & 39 (97.5) & $33(84.6)$ & $36(81.8)$ \\
\hline
\end{tabular}

$\mathrm{ND}=$ not done.

Study 1, a high number of ovulations were observed in women receiving a third cycle of treatment. As such, in Study 2, all women were treated for three cycles, and data were taken from Cycle 3 rather than from Cycle 1. A comparison of ovarian activity in Study 2 showed no difference between Cycles 2 and 3, consistent with previous findings [24].

In both studies, it was decided to consider a Hoogland score of 5 (LUF) to be as critical as a Hoogland score of 6 (ovulation), since the transition from a persisting FLS into a ruptured FLS might happen easily in subsequent cycles, as shown in Study 1. Spona et al. [25] administered a COC containing $20 \mathrm{mcg}$ of EE/100 mcg of levonorgestrel, and

Table 3

Frequently reported adverse events ( $\geq 5 \%$ of subjects in any regimen) considered to be (possibly/probably/definitely) related to treatment (FAS)

\begin{tabular}{llllll}
\hline $\begin{array}{l}\text { Adverse events } \\
{[n(\%)]}\end{array}$ & \multicolumn{3}{l}{ Study 1 } & & \multicolumn{2}{l}{ Study 2 } \\
\cline { 2 - 3 } \cline { 5 - 6 } & $\begin{array}{l}\text { Regimen 1A } \\
(n=96)\end{array}$ & $\begin{array}{l}\text { Regimen 2A } \\
(n=96)\end{array}$ & & $\begin{array}{l}\text { Regimen 2B } \\
(n=100)\end{array}$ & $\begin{array}{l}\text { Regimen 2C } \\
(n=103)\end{array}$ \\
\hline Headache & $16(16.7)$ & $13(13.5)$ & & $18(18.0)$ & $25(24.3)$ \\
Abdominal pain & $12(12.5)$ & $12(12.5)$ & & $12(12.0)$ & $11(10.7)$ \\
Acne & $7(7.3)$ & $7(7.3)$ & & $9(9.0)$ & $14(13.6)$ \\
Breast pain & $6(6.3)$ & $10(10.4)$ & & $6(6.0)$ & $10(9.7)$ \\
Dysmenorrhea & $6(6.3)$ & $4(4.2)$ & & $10(10.0)$ & $9(8.7)$ \\
Emotional & $5(5.2)$ & $1(1.0)$ & $7(7.0)$ & $10(9.7)$ \\
$\quad$ lability & & & & $7(7.0)$ & $6(5.8)$ \\
Nausea & $4(4.2)$ & $2(2.1)$ & & &
\end{tabular}

reported one LUF per 24 subjects in Cycle 2 and one LUF per 23 subjects in Cycle 3. This would have resulted in a point estimation of $4.2 \%$ and a $90 \%$ CI of $0.0-10.9 \%$. An ovulation rate of $<5 \%$ and an upper $90 \%$ CI of $<10 \%$ were defined in an effort to be 'on the safe side' before initiating a Phase III clinical trial program. Regimen $2 \mathrm{~B}$ reached this target with the lowest dose of DNG, whereas Regimen 2C reached a similar result but with a higher dose, which cannot be justified.

Hirvonen et al. [12] tested biphasic formulations of E2V plus either cyproterone acetate (CPA) or norethisterone (NET) in a total of 50 ovulating women over a period of 1 year. In total, 1 ovulatory cycle was observed in those women who received E2V/CPA (Cycle 1, $n=1$ ), compared with 11 ovulatory cycles in those women who received E2V/ NET (Cycle 1, $n=4$; Cycle 2, $n=2$; Cycle 3, $n=5$ ). However, it should be noted that women included in the study were aged 35-47 years and, therefore, were not in the optimal fertile age range. A follow-up study in 288 women showed an ovulation-inhibition rate of $95 \%$; however, the population had a mean age of $39.3 \pm 3.4$ years [11,13]. In addition, Csemiczky et al. [18] tested a biphasic regimen with E2 and desogestrel in 29 women with promising results in terms of ovulation inhibition and cycle control. However, no further data using this approach with bigger sample sizes have been identified.

The basis for the work described here, specifically Regimen 1A, was initially outlined by Hoffmann et al. $[14,19]$. They carried out a pilot study in 100 healthy women 
over six cycles, with no pregnancy and acceptable cycle control. Intermenstrual bleeding was recorded in $30 \%$ of cycles during the first three cycles. However, further (so far unpublished) data have revealed that ovulation inhibition was unsatisfactory with such a regimen, which led to the regimen modifications described in this article.

Currently available COCs have been proven to be safe and well tolerated in most women. Nevertheless, several attempts have been made to further improve the safety and tolerability profile of COCs. E2 is considerably less potent than $\mathrm{EE}$ in terms of hepatic protein synthesis induction, as demonstrated in clinical studies assessing sex-hormonebinding globulin, angiotensinogen and hemostatic parameters [26-29]. Such data suggest that a COC containing E2V at dosages used in the current study would induce less estrogen-related responses in the liver than currently available EE-containing COCs. However, further clinical and epidemiological studies will be needed to confirm whether this translates into better clinical outcomes.

Numerous attempts to replace EE with E2 in COCs have thus far been unsuccessful. In the studies described here, it was demonstrated that a high degree of ovulation inhibition can be achieved with a regimen containing E2V. The safety data of these two studies did not raise any concerns. The relatively high incidences of adverse events (Table 3) are likely due to the Phase II design, as seen in most studies of this type. Based on the findings of these studies, Regimen 2B was identified as the lowest effective dose for efficient suppression of ovulation. The cycle control, safety and efficacy of this regimen are currently being investigated in a Phase III clinical trial program. It is expected that this preparation will be the first globally available COC to use $\mathrm{E} 2$ instead of $\mathrm{EE}$ as the estrogen component, providing reliable contraceptive efficacy and acceptable cycle control.

\section{Acknowledgments}

The authors would like to thank Lyndal McMillan and Michael Klotzbücher for editorial assistance during the preparation of the manuscript.

\section{References}

[1] Dusterberg B, Ellman H, Muller U, Rowe E, Muhe B. A three-year clinical investigation into efficacy, cycle control and tolerability of a new low-dose monophasic oral contraceptive containing gestodene. Gynecol Endocrinol 1996;10:33-9.

[2] Huber J, Foidart JM, Wuttke W, et al. Efficacy and tolerability of a monophasic oral contraceptive containing ethinylestradiol and drospirenone. Eur J Contracept Reprod Health Care 2000;5: $25-34$.

[3] Spona J, Feichtinger W, Kindermann C, et al. Modulation of ovarian function by an oral contraceptive containing 30 micrograms ethinyl estradiol in combination with $2.00 \mathrm{mg}$ dienogest. Contraception 1997; 56:185-91.

[4] The safety and contraceptive efficacy of a 24-day low-dose oral contraceptive regimen containing gestodene 60 microg and ethinyles- tradiol 15 microg. Eur J Contracept Reprod Health Care 1999;4(Suppl 2):9-15.

[5] Bannemerschult R, Hanker JP, Wunsch C, Fox P, Albring M, Brill K. A multicenter, uncontrolled clinical investigation of the contraceptive efficacy, cycle control, and safety of a new low-dose oral contraceptive containing 20 micrograms ethinyl estradiol and 100 micrograms levonorgestrel over six treatment cycles. Contraception 1997;56: 285-90.

[6] Endrikat J, Jaques MA, Mayerhofer M, Pelissier C, Muller U, Dusterberg B. A twelve-month comparative clinical investigation of two low-dose oral contraceptives containing 20 micrograms ethinylestradiol/75 micrograms gestodene and 20 micrograms ethinylestradiol/150 micrograms desogestrel, with respect to efficacy, cycle control and tolerance. Contraception 1995;52:229-35.

[7] Nevinny-Stickel J. German trial of an oral contraceptive containing $0.150 \mathrm{mg}$ desogestrel plus $0.020 \mathrm{mg}$ ethinylestradiol. Acta Obstet Gynecol Scand 1987;144:19-21.

[8] World Health Organization Task Force on Oral Contraception. A randomized, double-blind study of two combined oral contraceptives containing the same progestogen, but different estrogens. Contraception 1980;21:445-59.

[9] Astedt B, Jeppsson S, Liedholm P, Rannevik G, Svanberg L. Clinical trial of a new oral contraceptive pill containing the natural oestrogen 17 beta-oestradiol. Br J Obstet Gynaecol 1979;86:732-6.

[10] Astedt B, Svanberg L, Jeppsson S, Liedholm P, Rannevik G. The natural oestrogenic hormone oestradiol as a new component of combined oral contraceptives. Br Med J 1977;1:269.

[11] Hirvonen E, Allonen H, Anttila M, et al. Oral contraceptive containing natural estradiol for premenopausal women. Maturitas 1995;21:27-32.

[12] Hirvonen E, Stenman UH, Malkonen M, Rasi V, Vartiainen E, Ylostalo P. New natural oestradiol/cyproterone acetate oral contraceptive for pre-menopausal women. Maturitas 1988;10:201-13.

[13] Hirvonen E, Vartiainen E, Kulmala Y, et al. A multicenter trial with a new OC using a natural estradiol and cyproterone acetate for women over 35. Adv Contracept 1990;6:248.

[14] Hoffmann H, Moore C, Kovacs L, et al. Alternatives of the replacement of ethinylestradiol by natural 17beta-estradiol in dienogest-containing oral contraceptives. Drugs Today 1999;35:105-13.

[15] Kivinen S, Saure A. Efficacy and tolerability of a combined oral contraceptive containing 17 beta-estradiol and desogestrel. Eur J Contracept Reprod Health Care 1996;1:183.

[16] Schubert W, Cullberg G. Ovulation inhibition with 17 beta-estradiol cyclo-octyl acetate and desogestrel. Acta Obstet Gynecol Scand 1987; 66:543-7.

[17] Serup J, Bostofte E, Larsen S, Westergaard J, Lebech PE. Natural oestrogens for oral contraception. Lancet 1979;2:471-2.

[18] Csemiczky G, Dieben T, Coeling Bennink HJ, Landgren BM. The pharmacodynamic effects of an oral contraceptive containing $3 \mathrm{mg}$ micronized 17 beta-estradiol and $0.150 \mathrm{mg}$ desogestrel for 21 days, followed by $0.030 \mathrm{mg}$ desogestrel only for 7 days. Contraception 1996; 54:333-8.

[19] Hoffmann H, Moore C, Zimmermann H, et al. Approaches to the replacement of ethinylestradiol by natural 17beta-estradiol in combined oral contraceptives. Exp Toxicol Pathol 1998;50:458-64.

[20] Oettel M, Carol W, Elger W, et al. A 19-norprogestin without 17-alphaethinyl group II: dienogest from a pharmacodynamic point of view. Drugs Today 1995;31:517-36.

[21] Timmer CJ, Geurts TB. Bioequivalence assessment of three different estradiol formulations in postmenopausal women in an open, randomized, single-dose, 3-way cross-over study. Eur J Drug Metab Pharmacokinet 1999;24:47-53.

[22] Hoogland HJ, Skouby SO. Ultrasound evaluation of ovarian activity under oral contraceptives. Contraception 1993;47:583-90.

[23] Benagiano G, Primiero FM, Farris M. Clinical profile of contraceptive progestins. Eur J Contracept Reprod Health Care 2004;9:182-93.

[24] Pierson RA, Archer DF, Moreau M, Shangold GA, Fisher AC, Creasy GW. Ortho Evra/Evra versus oral contraceptives: follicular 
development and ovulation in normal cycles and after an intentional dosing error. Fertil Steril 2003;80:34-42.

[25] Spona J, Feichtinger W, Kindermann C, Wunsch C, Brill K. Inhibition of ovulation by an oral contraceptive containing 100 micrograms levonorgestrel in combination with 20 micrograms ethinylestradiol. Contraception 1996;54:299-304.

[26] Mashchak CA, Lobo RA, Dozono-Takano R, et al. Comparison of pharmacodynamic properties of various estrogen formulations. Am J Obstet Gynecol 1982;144:511-8.
[27] Wiegratz I, Lee JH, Kutschera E, Winkler UH, Kuhl H. Effect of four oral contraceptives on hemostatic parameters. Contraception 2004;70: 97-106.

[28] Lindberg UB, Crona N, Stigendal L, Teger-Nilsson AC, Silfverstolpe G. A comparison between effects of estradiol valerate and low dose ethinyl estradiol on haemostasis parameters. Thromb Haemost 1989;61:65-9.

[29] Helgason S. Estrogen replacement therapy after the menopause. Estrogenicity and metabolic effects. Acta Obstet Gynecol Scand Suppl 1982;107:1-29. 